\title{
The fast image encryption algorithm based on substitution and diffusion
}

\author{
Yong Zhang ${ }^{1}$ and Xiaoyang Jia ${ }^{2}$ \\ ${ }^{1}$ School of Software and Communication Engineering, Jiangxi University of Finance and Economics \\ Nanchang, P. R. China \\ [e-mail: zhangyong@jxufe.edu.cn] \\ ${ }^{2}$ Changchun China Optical Science \& Technology Museum \\ Changchun, P.R. China \\ [e-mail: jiaxiaoyang_cc@126.com] \\ *Corresponding author: Yong Zhang
}

Received December 27, 2017; revised February 19, 2018; accepted May 8, 2018;

published September 30, 2018

\begin{abstract}
A fast image encryption system based on substitution and diffusion was proposed, which includes one covering process, one substitution process and two diffusion processes. At first, Chen's chaotic system together with an external 256-bit long secret key was used to generate the key streams for image encryption, in which the initial values of Chen's chaotic system were regarded as the public key. Then the plain image was masked by the covering process. After that the resulting image was substituted with the disturbed S-Box of AES. Finally, the substituted image was diffused twice with the add-modulo operations as the core to obtain the cipher image. Simulation analysis and comparison results with AES and some existing image cryptosystems show that the proposed image cryptosystem possesses the merits of fast encryption/decryption speed, good statistical characteristics, strong sensitivity and etc., and can be used as a candidate system of network security communication.
\end{abstract}

Keywords: Information security; image encryption; chaos; substitution; diffusion 


\section{Introduction}

Chaotic image cryptology research has become a hotspot in the field of information security. And that includes three main research directions: First is to study the new chaotic systems which can produce cryptographic key streams [1-5]. The direction serves as an important application of chaos theory to give birth to the study of new chaotic systems. Second is to study the new image encryption schemes [6-10]. The research results of this direction show that the variety of novel image confusion and diffusion algorithms has emerged in recent decades. Third is to study the crypto-analysis of image cryptosystem [11-15]. Accompanied by the emergence of a large number of image encryption systems, quantities of crypto-analysis systems have been proposed, which are simulating the further research on new high-intensity image cryptography.

Recently, Zhang and Tang suggested an image cryptosystem with identical encryption and decryption algorithm [16]. The length of secret key is 64d bits (where, $d$ is a positive integer). The encryption/decryption process consists of two diffusion operations, two matrix rotations and one plaintext-related scrambling. Due to skillful using the matrix rotations, the encryption process and decryption process in their scheme share the same algorithm. Chai et al. proposed an image encryption system using the memristive hyper-chaotic system, DNA encoding and cellular automaton [17]. The system employed the SHA-256 Hash value of plain image as the secret key of size 256 bits. The secret key was then converted into four initial values of memristive hyper-chaotic system to iteratively generate the key streams. Finally, the key streams were used in confusion and diffusion processes under the rules of DNA encoding and cellular automaton. From the point of view of cryptography, Chai et al.'s system is essentially a complex one-time pad system, which has certain theoretical research value, but almost no application value. Diaconu presented a new image encryption system using bit-level permutation [18]. In this system each row of image was converted into a bit sequence, and the bit-shift operation was based on the whole row of bit sequence. This algorithm is very suitable for hardware implementation, but not suitable for computer software implementation. At the same time, Fan et al. cracked this image encryption system by means of chosen plaintext attack [19]. Zhang et al. delivered an image encryption algorithm with typical confusion-diffusion architecture based on Chen's chaotic system and 3D Cat map [20]. In order to improve the processing speed of encryption, their scheme used 3D bit permutation algorithm based on sorting method and 3D Cat transform matrix. However, Zhang et al. pointed out that the system in [20] cannot frustrate the chosen plaintext attack [21].

All of the aforesaid image encryption systems used the confusion-diffusion architecture. In this architecture the diffusion algorithms usually employ add-then-modulo operation or XOR operation, while the confusion algorithms are the main innovation points of cryptographic scheme [22]. However, some of the research results have obvious security 
vulnerabilities, so as to fail in confronting the chosen/known plaintext attacks $[19,21]$. Aiming at reducing the above security problems as much as possible, this paper proposed a novel image cryptosystem based on the combination of substitution and diffusion algorithms. The substitution technology instead of confusion is used for image information hiding, hoping to get better information security performance. This paper starts with the research on key streams generator with an external key and the Chen's chaotic system. The initial values of Chen's system are taken as the public key. Then, the key streams are used to cover the plain image. After that the covered image is encrypted into cipher image by means of substitution-diffusion-diffusion architecture. Finally, the simulation test and theoretical analysis are performed to verify that the proposed image cryptosystem possesses good security characteristics and fast encryption/decryption speed.

The rest of this paper is organized as follows: Section 2 introduces the chaotic pseudo random sequence generator; Section 3 details the structure of proposed image cryptosystem and its algorithm implementation steps; Section 4 gives the typical representatives of simulation results; Section 5 performs the security performance analyses of proposed image cryptosystem, and compares them with some of existing image cryptosystems; Finally, Section 6 summarizes the full paper.

\section{Pseudo random sequence generator}

Chaotic systems are widely used to generate pseudo random sequences due to their extreme sensitivity to initial values and parameters, and ergodicity of state space. This section presents a method for generating pseudo random sequences by multiple initial states acting on a chaotic system. Without losing generality, take a one-dimensional chaotic system as an example. Assume that the discrete state evolution equation of one-dimensional chaotic system is described by

$$
x(n)=F(x(n-1) ; \boldsymbol{u})
$$

Where, $\boldsymbol{u}$ is the parameter vector, and $x(n)$ is the $n$-th state.

Now, let there be a total of $k$ pieces of initial states, denoted by $\left\{s_{i}\right\}, i=1,2, \ldots, k$. The steps of generating pseudo random sequences are as follows:

Step 1. Randomly take a state value $x_{0}$ from the state space of Eq. (1) as the initial value of Eq. (1), then iterate it for $l$ times $(l>10)$. Denote the last 10 iteration values as $\left\{X_{l-9,}, X_{l-8}, X_{l-7}, \ldots, X_{l-1}, X_{l}\right\}$, and then calculate $\lambda$ by the following formula

$$
\lambda=\frac{\left|x_{l}\right|}{\sum_{j=0}^{9}\left|x_{l-j}\right|}
$$

Where, $|x|$ returns the absolute value of $x$.

Step 2. Let $i=1$.

Step 3. Let new $x_{l}=(1-\lambda) x_{l}+\lambda s_{i}$. Take the new $x_{l}$ as the initial value $x_{0}$ of Eq. (1), and then iterate it for $l$ times. Denote the last 10 iteration values as $\left\{X_{l-9,}, X_{l-8}, X_{l-7}, \ldots, X_{l-1}, X_{l}\right\}$, and then 
calculate the new $\lambda$ also by Eq. (2).

Step 4. Let $i=i+1$. If $i<k$, then jump to Step 3; otherwise, continue to Step 5.

Step 5. Let new $x_{l}=(1-\lambda) x_{l}+\lambda s_{k}$. Take the new $x_{l}$ as the initial value $x_{0}$ of Eq. (1), and then iterate it for $l$ times to bypass the transient state. Then, continue to iterate Eq. (1) for $L$ times to get a floating-point state sequence of length $L$, denoted by $\boldsymbol{x}=\left\{x_{j}, j=1,2, \ldots, L\right\}$.

Step 6. Convert the sequence $\boldsymbol{x}$ into an integer sequence, denoted by $\boldsymbol{t}=\left\{t_{j}, j=1,2, \ldots, L\right\}$, using the following formula.

$$
t_{j}=\left(\left|x_{j}\right| \times 2^{32}\right) \bmod 256, j=1,2, \ldots, L
$$

And the sequence $\boldsymbol{t}$ is the key stream for image encryption.

For a multidimensional chaotic system, one can choose an optional state form the state vector of discretized state equation as the role of the above $x$ of one-dimensional chaotic system. Then, one can use the above method to generate pseudo random sequence by multiple initial states acting on the multidimensional chaotic system.

The proposed image cryptosystem is as shown in Fig. 1. In Fig. 1, three pseudo random matrices $\boldsymbol{X}, \boldsymbol{Y}$ and $\boldsymbol{Z}$, and three pseudo random numbers $r_{1}, r_{2}$ and $r_{3}$, are generated by the secret key $\boldsymbol{K}$ with the help of key stream generator. Here, the secret key $\boldsymbol{K}$ used is of length 256 bits and consists of 32 pieces of 8-bit unsigned bytes, namely, $K=\left\{K_{i}, i=1,2, \ldots, 32\right\}$, where, each $K_{i}$ is a byte. The $\boldsymbol{K}$ is transformed into 32 pieces of initial states, denoted by $\boldsymbol{s}=\left\{s_{i}, i=1,2, \ldots, 32\right\}$, using the following formula.

$$
s_{i}=K_{i} / 256, i=1,2, \ldots, 32
$$

\begin{tabular}{|c|c|c|c|c|c|c|c|c|c|}
\hline $\begin{array}{c}\text { Plain image } \\
\boldsymbol{P}_{M \times N} \\
\end{array}$ & \multicolumn{2}{|c|}{$\begin{array}{l}\text { Covering } \\
\text { operation }\end{array}$} & $\underset{M \times N}{\longrightarrow}$ & Substitution & $\stackrel{\boldsymbol{B}_{M \times N}}{\longrightarrow}$ & Diffusion-I & $\stackrel{\boldsymbol{D}_{M \times N}}{\longrightarrow}$ & Diffusion-II & \multirow[t]{2}{*}{$\begin{array}{c}\text { Cipher image } \\
\boldsymbol{C}_{M \times N} \\
\end{array}$} \\
\hline Encryption $\mathrm{p}$ & & $\boldsymbol{X}_{M \times N}$ & & $r_{1}, r_{2}$ & & $\boldsymbol{Y}_{M \times N}$ & \multicolumn{2}{|c|}{$Z_{M \times N}, r_{3}$} & \\
\hline $\begin{array}{c}\text { Secret key } \\
\boldsymbol{K}\end{array}$ & \multicolumn{8}{|c|}{ Key stream generator $\left(\boldsymbol{X}_{M \times N}, \boldsymbol{Y}_{M \times N}, \boldsymbol{Z}_{M \times N}, r_{1}, r_{2}, r_{3}\right)$} & \\
\hline Decryption $\mathrm{p}$ & \multicolumn{3}{|c|}{$Z_{M \times N}, r_{3}$} & $\boldsymbol{Y}_{M \times N}$ & & $r_{1}, r_{2}$ & \multicolumn{2}{|r|}{$\boldsymbol{X}_{M \times N}$} & \\
\hline $\begin{array}{c}\text { Cipher image } \\
C_{M \times N}\end{array}$ & & $\begin{array}{l}\text { erse of } \\
\text { usion-II }\end{array}$ & & $\begin{array}{l}\text { Inverse of } \\
\text { Diffusion-I }\end{array}$ & & $\begin{array}{c}\text { Inverse of } \\
\text { Substitution }\end{array}$ & & $\begin{array}{l}\text { Uncovering } \\
\text { operation }\end{array}$ & $\begin{array}{c}\text { Plain image } \\
\boldsymbol{P}_{M \times N}\end{array}$ \\
\hline
\end{tabular}

Fig. 1. Proposed image cryptosystem

Any chaotic system that can generate cryptographic pseudo random numbers can be chosen as the mapping $F$ in Eq. (1). Here, Chen's chaotic system [23] is selected, and its formula is as follows.

$$
\left\{\begin{array}{c}
\dot{x}=a(y-x) \\
\dot{y}=(c-a) x-x z+c y \\
\dot{z}=x y-b z
\end{array}\right.
$$

Where, $a=35, b=3, c \in[20,28.4]$. When $c=28$ (used in this paper), the phase portrait of Chen's system is as shown in Fig. 2 (with the size of discretized step being 0.002). 


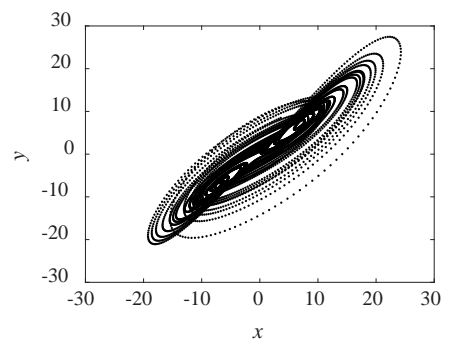

(a) $x$ vs. $y$

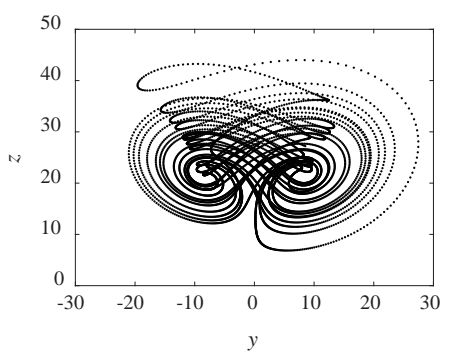

(b) $y$ vs. $z$

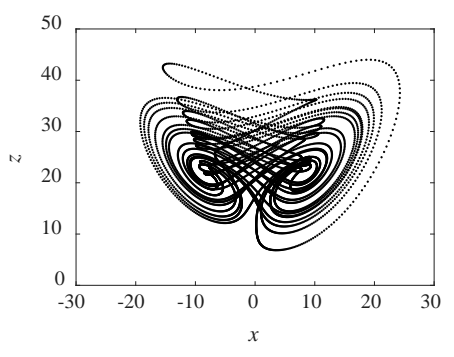

(c) $X$ VS. Z

Fig. 2. Phase portraits of Chen's system.

Randomly select a group of state values $\left(x_{0}, y_{0}, z_{0}\right)$ from the state space of Eq. (5) as the initial values of iteration (which are open and serve as public key). Here, let $l=100$. Assume that the plain image is of size $M \times N$, and the state $x$ is selected as the disturbed state. Employ the above method with multiple initial states $s$ and $\left(x_{0}, y_{0}, z_{0}\right)$ acting on Chen's system to iteratively generate three chaotic state sequences $\left\{x_{i}\right\},\left\{y_{i}\right\}$ and $\left\{z_{i}\right\}$ all of length $L / 3$, where, $L=3 M N+3$. Then, convert these three sequences into an integer sequence $\boldsymbol{t}=\left\{t_{i}\right\}$ of length $L=3 M N+3$ using the following formulae.

$$
t_{3 j-2}=\left[\left|x_{j}\right| \times 2^{32}\right] \bmod 256, t_{3 j-1}=\left[\left|y_{j}\right| \times 2^{32}\right] \bmod 256, t_{3 j}=\left[\left|z_{j}\right| \times 2^{32}\right] \bmod 256
$$

Where, $j=1,2, \ldots, L / 3$, and $[x]$ returns the integer part of $x$.

Then, generate $\boldsymbol{X}, \boldsymbol{Y}, \boldsymbol{Z}, r_{1}, r_{2}$ and $r_{3}$ form the sequence $\boldsymbol{t}$ with the following formulae.

$$
\begin{aligned}
& \boldsymbol{X}(i, j)=t_{N(i-1)+j}, \boldsymbol{Y}(i, j)=t_{M N+N(i-1)+j}, \boldsymbol{Z}(i, j)=t_{2 M N+N(i-1)+j}, i=1,2, \ldots, M, j=1,2, \ldots, N \\
& r_{1}=t_{3 M N+1}, r_{2}=t_{3 M N+2}, r_{3}=t_{3 M N+3}
\end{aligned}
$$

The matrices $\boldsymbol{X}, \boldsymbol{Y}$ and $\boldsymbol{Z}$ and the pseudo random number $r_{1}, r_{2}$ and $r_{3}$ are the key streams of proposed image cryptosystem.

To prove that the sequence $\boldsymbol{t}$ possesses good cryptographic characteristics, the test items of FIPS140-2 [24], such as Monobit test, Poker test, Runs test and Long run test, are performed on the sequence $\boldsymbol{t}$. Without loss of generality, assume that the initial values of Chen's system are $\left(x_{0}, y_{0}, z_{0}\right)=(0.56,0.809,12.347)$, the step size is $0.002, l=100$, and the secret key $\boldsymbol{K}=\{65,212,155,202,4,27,202,161,7,233,146,36,229,154,157,109,193$, $182,117,206,39,244,177,216,34,255,74,55,12,223,205,112\}$ (in decimal format). By the above method a sequence $\boldsymbol{t}$ of length 2500 bytes is obtained. Then, the sequence $\boldsymbol{t}$ is

\begin{tabular}{|c|c|c|c|c|c|c|c|c|c|}
\hline \multirow{3}{*}{ Test item } & \multirow{3}{*}{$\begin{array}{c}\text { Monobit } \\
\text { test }\end{array}$} & \multirow{3}{*}{$\begin{array}{c}\text { Poker } \\
\text { test }\end{array}$} & \multicolumn{6}{|c|}{ Runs test } & \multirow{3}{*}{$\begin{array}{l}\text { Long } \\
\text { run test } \\
(>25)\end{array}$} \\
\hline & & & \multicolumn{6}{|c|}{ Length of runs } & \\
\hline & & & 1 & 2 & 3 & 4 & 5 & $>5$ & \\
\hline bit 0 & 9878 & 100 & 2538 & 1206 & 614 & 327 & 153 & 145 & 0 \\
\hline bit 1 & 10122 & & 2419 & 1291 & 617 & 320 & 170 & 166 & 0 \\
\hline
\end{tabular}
converted into a bit sequence of length 20000 bits, whose test results are listed in Table 1.

Table 1. FIPS140-2 test results of sequence $t$ 


\begin{tabular}{|c|c|c|c|c|c|c|c|c|c|}
\hline $\begin{array}{c}\text { Theoretical } \\
\text { value }\end{array}$ & $9725 \sim$ & $2.16 \sim$ & $2315 \sim$ & $1114 \sim$ & $527 \sim$ & $240 \sim$ & $103 \sim$ & $103 \sim$ & 0 \\
& 10725 & 46.17 & 2685 & 1386 & 723 & 384 & 209 & 209 & 09 \\
\hline Results & Pass & Pass & Pass & Pass & Pass & Pass & Pass & Pass & Pass \\
\hline
\end{tabular}

As can be seen from Table 1, the sequence $\boldsymbol{t}$ passes all the test items of FIPS140-2, which shows that the proposed pseudo random number generator can produce cryptographic pseudo random sequences with good statistical characteristics.

The following analyzes the autocorrelation of the sequence $\boldsymbol{t}$. Convert $\boldsymbol{t}$ into a sequence of zero mean, denoted by $s$, with the following formula.

$$
\boldsymbol{s}(i)=2 \boldsymbol{t}(i)-255, i=1,2, \ldots, L
$$

Where, $L=12000$, and $\boldsymbol{t}$ is generated by the above method with the same initial values and secret key $\boldsymbol{K}$. Then, calculate the cyclic autocorrelation of $\boldsymbol{s}$ and illustrate the result of correlation coefficient in Fig. 3.

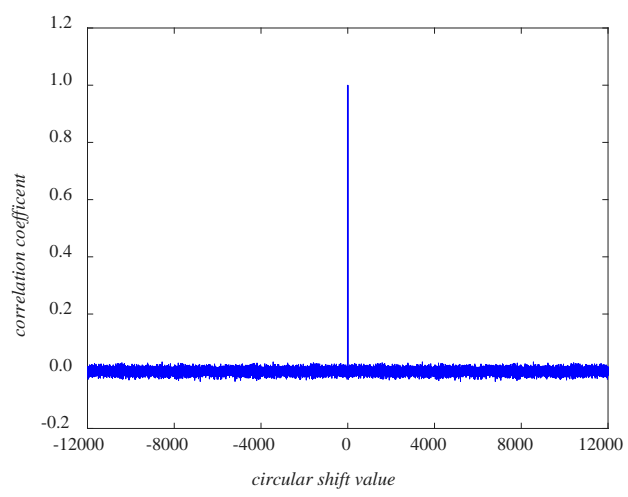

Fig. 3. Cyclic autocorrelation of sequence $s$

It can be seen from Fig. 3 that the correlation coefficient curve of the sequence $\boldsymbol{s}$ is thumbtack-like, which shows that the sequence $\boldsymbol{s}$ (or its predecessor sequence $\boldsymbol{t}$ ) has no correlation between the cyclic-shift sequences. So the adjacent data in the sequence $\boldsymbol{t}$ are not predictable, i.e. the sequence $\boldsymbol{t}$ can be used as the key stream for image encryption.

\section{Image cryptosystem}

In the proposed image cryptosystem shown in Fig. 1, the encryption process includes one covering operation, one substitution process and two diffusion processes. The decryption process is the inverse of the encryption process. Both the encryption process and the decryption process have no permutation or confusion process. 
In Fig. 1, the plain image $\boldsymbol{P}$ is assumed to be an 8-bit grayscale image of size $M \times N$, and the secret key $\boldsymbol{K}$ is of length 256 bits. The matrices $\boldsymbol{X}, \boldsymbol{Y}$ and $\boldsymbol{Z}$ and the three pseudo random numbers $r_{1}, r_{2}$ and $r_{3}$ used as the key streams in encryption/decryption process are generated by the method described in Section 2 with the secret key $\boldsymbol{K}$. The details of encryption and decryption processes are as follows.

\section{(1) Covering operation and its inverse}

From Fig. 1, the covering operation converts the plain image $\boldsymbol{P}$ into a matrix, denoted by $A$, using the following formula.

$$
\boldsymbol{A}(i, j)=\boldsymbol{P}(i, j) \oplus \boldsymbol{X}(i, j), i=1,2, \ldots, M, j=1,2, \ldots, N
$$

Where, $\oplus$ represents bitwise XOR (exclusive or) operation (the same meaning thereinafter). The covering operation uses the matrix $\boldsymbol{X}$ to mask the plain image $\boldsymbol{P}$ with the XOR operation. Because the XOR operation is invertible, the inverse of Eq. (10) is as follows.

$$
\boldsymbol{P}(i, j)=\boldsymbol{A}(i, j) \oplus \boldsymbol{X}(i, j), i=1,2, \ldots, M, j=1,2, \ldots, N
$$

From Eq. (11), one can recover $\boldsymbol{P}$ from $\boldsymbol{A}$ with the help of $\boldsymbol{X}$.

\section{(2) Substitution process and its inverse}

The S-Box of AES [25] shown in Table 2 is used in the substitution process.

For any 8-bit byte $a$, its higher 4 bits are considered as $x$, and the lower 4 bits as $y$, then one can get a unique 8-bit byte $b$ by looking up the Table 2 with the coordinates $(x, y)$, which is denoted by

$$
a \stackrel{\text { S-Box }}{\longrightarrow} b
$$

The concrete steps of substitution are as follows.

Step 1. XOR each element of S-Box in Table 2 with $r_{1}$ to get a new S-Box. The new S-Box is employed in the following steps.

Step 2. Let $\boldsymbol{T}(1,1)=\left[\boldsymbol{A}(1,1)+r_{2}\right]$ mod 256 , and obtain $\boldsymbol{B}(1,1)$ from $\boldsymbol{T}(1,1)$ by looking up the $\mathrm{S}$-Box. This process is denoted by

$$
\left[\boldsymbol{A}(1,1)+r_{2}\right] \bmod 256=\boldsymbol{T}(1,1) \stackrel{\text { S-Box }}{\longrightarrow} \boldsymbol{B}(1,1)
$$

Step 3. Let $j=2$ to $N$, then perform the following for each $j$.

$$
[\boldsymbol{A}(1, j)+\boldsymbol{A}(1, j-1)+\boldsymbol{T}(1, j-1)] \bmod 256=\boldsymbol{T}(1, j) \stackrel{\text { S-Box }}{\longrightarrow} \boldsymbol{B}(1, j)
$$

Step 4. Replace $\boldsymbol{A}(2,1)$ by $\boldsymbol{B}(2,1)$ with the following formula.

$$
[\boldsymbol{A}(2,1)+\boldsymbol{A}(1,1)+\boldsymbol{A}(1, \mathrm{~N})+\boldsymbol{T}(1,1)+\boldsymbol{T}(1, N)] \bmod 256=\boldsymbol{T}(2,1) \stackrel{\text { s-Box }}{\longrightarrow} \boldsymbol{B}(2,1)
$$

Step 5. Let $i=3$ to $M$, then do the following for each $i$.

$$
[A(i, 1)+A(i-1,1)+\boldsymbol{T}(i-1,1)] \bmod 256=\boldsymbol{T}(i, 1) \stackrel{\text { s-Box }}{\longrightarrow} \boldsymbol{B}(i, 1)
$$


Table 2. S-Box of AES

\begin{tabular}{|c|c|c|c|c|c|c|c|c|c|c|c|c|c|c|c|c|c|}
\hline \multirow{2}{*}{\multicolumn{2}{|c|}{ S-Box }} & \multicolumn{16}{|c|}{$y$} \\
\hline & & \multirow{2}{*}{$\begin{array}{c}0 \\
99\end{array}$} & \multirow{2}{*}{$\frac{1}{124}$} & \multirow{2}{*}{$\begin{array}{c}2 \\
119\end{array}$} & \multirow{2}{*}{$\begin{array}{c}3 \\
123\end{array}$} & \multirow{2}{*}{$\begin{array}{c}4 \\
242\end{array}$} & \multirow{2}{*}{$\begin{array}{c}5 \\
107\end{array}$} & \multirow{2}{*}{$\frac{6}{111}$} & \multirow{2}{*}{$\begin{array}{c}7 \\
197\end{array}$} & \multirow{2}{*}{$\begin{array}{c}8 \\
48\end{array}$} & \multirow{2}{*}{$\begin{array}{l}9 \\
1\end{array}$} & \multirow{2}{*}{$\begin{array}{c}10 \\
103\end{array}$} & \multirow{2}{*}{$\frac{11}{43}$} & \multirow{2}{*}{$\frac{12}{254}$} & \multirow{2}{*}{$\begin{array}{c}13 \\
215\end{array}$} & \multirow{2}{*}{$\begin{array}{c}14 \\
171\end{array}$} & \multirow{2}{*}{$\begin{array}{c}15 \\
118\end{array}$} \\
\hline & 0 & & & & & & & & & & & & & & & & \\
\hline & 1 & 202 & 130 & 201 & 125 & 250 & 89 & 71 & 240 & 173 & 212 & 162 & 175 & 156 & 164 & 114 & 192 \\
\hline & 2 & 183 & 253 & 147 & 38 & 54 & 63 & 247 & 204 & 52 & 165 & 229 & 241 & 113 & 216 & 49 & 21 \\
\hline & 3 & 4 & 199 & 35 & 195 & 24 & 150 & 5 & 154 & 7 & 18 & 128 & 226 & 235 & 39 & 178 & 117 \\
\hline & 4 & 9 & 131 & 44 & 26 & 27 & 110 & 90 & 160 & 82 & 59 & 214 & 179 & 41 & 227 & 47 & 132 \\
\hline & 5 & 83 & 209 & 0 & 237 & 32 & 252 & 177 & 91 & 106 & 203 & 190 & 57 & 74 & 76 & 88 & 207 \\
\hline & 6 & 208 & 239 & 170 & 251 & 67 & 77 & 51 & 133 & 69 & 249 & 2 & 127 & 80 & 60 & 159 & 168 \\
\hline & 7 & 81 & 163 & 64 & 143 & 146 & 157 & 56 & 245 & 188 & 182 & 218 & 33 & 16 & 255 & 243 & 210 \\
\hline \multirow{8}{*}{$x$} & 8 & 205 & 12 & 19 & 236 & 95 & 151 & 68 & 23 & 196 & 167 & 126 & 61 & 100 & 93 & 25 & 115 \\
\hline & 9 & 96 & 129 & 79 & 220 & 34 & 42 & 144 & 136 & 70 & 238 & 184 & 20 & 222 & 94 & 11 & 219 \\
\hline & 10 & 224 & 50 & 58 & 10 & 73 & 6 & 36 & 92 & 194 & 211 & 172 & 98 & 145 & 149 & 228 & 121 \\
\hline & 11 & 231 & 200 & 55 & 109 & 141 & 213 & 78 & 169 & 108 & 86 & 244 & 234 & 101 & 122 & 174 & 8 \\
\hline & 12 & 186 & 120 & 37 & 46 & 28 & 166 & 180 & 198 & 232 & 221 & 116 & 31 & 75 & 189 & 139 & 138 \\
\hline & 13 & 112 & 62 & 181 & 102 & 72 & 3 & 246 & 14 & 97 & 53 & 87 & 185 & 134 & 193 & 29 & 158 \\
\hline & 14 & 225 & 248 & 152 & 17 & 105 & 217 & 142 & 148 & 155 & 30 & 135 & 233 & 206 & 85 & 40 & 223 \\
\hline & 15 & 140 & 161 & 137 & 13 & 191 & 230 & 66 & 104 & 65 & 153 & 45 & 15 & 176 & 84 & 187 & 22 \\
\hline
\end{tabular}

Step 6. Let $i=2$ to $M$ and $j=2$ to $N$, then do the following for each combination of $i$ and $j$.

$$
[\boldsymbol{A}(i, j)+\boldsymbol{A}(i, j-1)+\boldsymbol{A}(i-1, j)+\boldsymbol{T}(i, j-1)+\boldsymbol{T}(i-1, j)] \bmod 256=\boldsymbol{T}(i, j) \stackrel{\text { s-Box }}{\longrightarrow} \boldsymbol{B}(i, j)
$$

After the above processing, the matrix $\boldsymbol{A}$ is replaced by the matrix $\boldsymbol{B}$ with the help of $r_{1}$, $r_{2}$ and S-Box.

The above substitution process is reversible, and the matrix $\boldsymbol{A}$ can be recovered from the matrix $\boldsymbol{B}$ with the help of $r_{1}, r_{2}$ and S-Box. The inverse of the substitution is as follows:

Step 1. Denote the inverse of S-Box in Table 2 by S-Box ${ }^{-1}$.

Step 2. XOR each element of $\boldsymbol{B}$ with $r_{1}$ to get a new matrix, denoted by $\boldsymbol{G}$.

Step 3. Obtain $\boldsymbol{T}(1,1)$ from $\boldsymbol{G}(1,1)$ by looking up S-Box ${ }^{-1}$, then get $\boldsymbol{A}(1,1)$ by calculating $\left[\boldsymbol{T}(1,1)-r_{2}+256\right] \bmod 256$. This process is denoted by

$$
\boldsymbol{G}(1,1) \stackrel{\text { S-Box }}{\longrightarrow} T(1,1) \text {, then }\left[\boldsymbol{T}(1,1)+256-r_{2}\right] \bmod 256=\boldsymbol{A}(1,1)
$$

Step 4. Let $j=2$ to $N$, then carry out the following formula for each $j$.

$$
\boldsymbol{G}(1, j) \stackrel{\text { S-Box }}{\longrightarrow} \boldsymbol{T}(1, j) \text {, then }[\boldsymbol{T}(1, j)+256 \times 2-\boldsymbol{T}(1, j-1)-\boldsymbol{A}(1, j-1)] \bmod 256=\boldsymbol{A}(1, j)
$$

Step 5. Recover $\boldsymbol{A}(2,1)$ from $\boldsymbol{G}(2,1)$ by the following formula.

$\boldsymbol{G}(2,1) \stackrel{\text { S-Box }}{\longrightarrow} \boldsymbol{T}(2,1)$, then $[\boldsymbol{T}(2,1)+256 \times 4-\boldsymbol{T}(1,1)-\boldsymbol{T}(1, N)-\boldsymbol{A}(1,1)-\boldsymbol{A}(1, N)]$ mod 256=A(2,1) 
Step 6. Let $i=3$ to $M$, then do the following formula for each $i$.

$$
\boldsymbol{G}(i, 1) \stackrel{{\mathrm{S}-\mathrm{Box}^{-1}}^{\longrightarrow}}{\longrightarrow} \boldsymbol{T}(i, 1) \text {, then }[\boldsymbol{T}(i, 1)+256 \times 2-\boldsymbol{T}(i-1,1)-\boldsymbol{A}(i-1,1)] \bmod 256=\boldsymbol{A}(i, 1)
$$

Step 7. Let $i=2$ to $M$, and $j=2$ to $N$, then do the following formula for each combination of $i$ and $j$.

$\boldsymbol{G}(i, j) \stackrel{\text { s-Box }}{\longrightarrow} \boldsymbol{T}(i, j)$, then $[\boldsymbol{T}(i, j)+256 \times 4-\boldsymbol{T}(i, j-1)-\boldsymbol{T}(i-1, j)-\boldsymbol{A}(i, j-1)-\boldsymbol{A}(i-1, j)] \bmod 256=\boldsymbol{A}(i, j)$

After the above processing, the matrix $\boldsymbol{A}$ is recovered from the matrix $\boldsymbol{B}$ with the help of $r_{1}, r_{2}$ and S-Box.

\section{(3) Diffusion-I process and its inverse}

The diffusion process disperses the information of each pixel in the original image to as many pixels as possible to get the resultant image. The multiplication based on Galois field $\mathrm{GF}\left(2^{8}\right)$ is used in the diffusion process with the primitive polynomial being $m(x)=x^{8}+x^{4}+x^{3}+x+1$. In Fig. 1, the diffusion process includes two stages, namely, the diffusion-I and the diffusion-II. The latter will be discussed in the next part labeled with (4) of subhead. The specific steps of diffusion-I process are as follows:

Step 1. Obtain $\boldsymbol{D}(M, N)$ from $\boldsymbol{B}(M, N)$ and $\boldsymbol{Y}(M, N)$ using the following

$$
\boldsymbol{D}(M, N)=[\boldsymbol{B}(M, N)+\boldsymbol{Y}(M, N)] \bmod 256
$$

Step 2. Let $j=N-1$ to 1 , then calculate $D(M, j)$ for each $j$ with the following formula.

$$
\boldsymbol{D}(M, j)=[\boldsymbol{B}(M, j)+\boldsymbol{B}(M, j+1)+\boldsymbol{D}(M, j+1)+\boldsymbol{Y}(M, j)] \bmod 256
$$

Step 3. Generate $\boldsymbol{D}(M-1, N)$ from $\boldsymbol{B}(M-1, N), \mathbf{Y}(M-1, N), \boldsymbol{B}(M, 1)$ and $\boldsymbol{D}(M, 1)$ with the following formula.

$$
\boldsymbol{D}(M-1, N)=[\boldsymbol{B}(M-1, N)+\mathrm{B}(\mathrm{M}, 1)+\boldsymbol{D}(M, 1)+\boldsymbol{Y}(M-1, N)] \bmod 256
$$

Step 4. Let $i=M-2$ to 1 , then calculate $\boldsymbol{D}(i, N)$ for each $i$ with the following formula.

$$
\boldsymbol{D}(i, N)=[\boldsymbol{B}(i, N)+\boldsymbol{B}(i+1, N)+\boldsymbol{D}(i+1, N)+\boldsymbol{Y}(i, N)] \bmod 256
$$

Step 5. Let $i=M-1$ to 1 , and $j=N-1$ to 1 , then calculate $\boldsymbol{D}(i, j)$ for each combination of $i$ and $j$ with the following formula.

$\boldsymbol{D}(i, j)=\{\boldsymbol{B}(i, j)+\boldsymbol{Y}(i, j) \cdot[\boldsymbol{D}(i, j+1) \oplus \boldsymbol{B}(i+1, j)]+[\boldsymbol{Y}(i, j) \oplus 128] \cdot[\boldsymbol{D}(i+1, j) \oplus \boldsymbol{B}(i, j+1)]\} \bmod 256$

After the above processing, one can get the matrix $\boldsymbol{D}$ from the matrices $\boldsymbol{B}$ and $\boldsymbol{Y}$.

The diffusion-I process is reversible, and its inverse process recovers $\boldsymbol{B}$ from $\boldsymbol{D}$ and $\boldsymbol{Y}$ with the following steps:

Step 1. Obtain $\boldsymbol{B}(M, N)$ from $\boldsymbol{D}(M, N)$ and $\boldsymbol{Y}(M, N)$ with the following formula.

$$
\boldsymbol{B}(M, N)=[\boldsymbol{D}(M, N)+256-\boldsymbol{Y}(M, N)] \bmod 256
$$

Step 2. Let $j=N-1$ to 1 , then calculate $\boldsymbol{B}(M, j)$ for each $j$ with the following formula.

$$
\boldsymbol{B}(M, j)=[\boldsymbol{D}(M, j)+256 \times 3-\boldsymbol{D}(M, j+1)-\mathrm{B}(\mathrm{M}, \mathrm{j}+1)-\boldsymbol{Y}(M, j)] \bmod 256
$$

Step 3. Generate $\boldsymbol{B}(M-1, N)$ from $\boldsymbol{D}(M-1, N), \boldsymbol{D}(M, 1), \boldsymbol{Y}(M-1, N)$ and $\boldsymbol{B}(M, 1)$ with the following formula.

$\boldsymbol{B}(M-1, N)=[\boldsymbol{D}(M-1, N)+256 \times 3-\boldsymbol{D}(M, 1)-\mathrm{B}(\mathrm{M}, 1)-\boldsymbol{Y}(M-1, N)] \bmod 256$ 
Step 4. Let $i=M-2$ to 1 , then calculate $\boldsymbol{B}(i, N)$ for each $i$ with the following formula.

$$
\boldsymbol{B}(i, N)=[\boldsymbol{D}(i, N)+256 \times 3-\boldsymbol{D}(i+1, N)-\mathrm{B}(\mathrm{i}+1, \mathrm{~N})-\boldsymbol{Y}(i, N)] \bmod 256
$$

Step 5. Let $i=M-1$ to 1 , and $j=N-1$ to 1 , then calculate $\boldsymbol{B}(i, j)$ for each combination of $i$ and $j$ with the following formula.

$\boldsymbol{B}(i, j)=\{\boldsymbol{D}(i, j)+256 \times 4-\boldsymbol{Y}(i, j) \cdot[\boldsymbol{D}(i, j+1) \oplus \boldsymbol{B}(i+1, j)]-[\boldsymbol{Y}(i, j) \oplus 128] \cdot[\boldsymbol{D}(i+1, j) \oplus \boldsymbol{B}(i, j+1)]\} \bmod 256$

After the above processing, one can recover $\boldsymbol{B}$ from $\boldsymbol{D}$ and $\boldsymbol{Y}$.

\section{(4) Diffusion-II process and its inverse}

From Fig. 1, the diffusion-II produces the cipher image $\boldsymbol{C}$ from the matrices $\boldsymbol{D}, \boldsymbol{Z}$ and $r_{3}$. The concrete steps of diffusion-II are as follows.

Step 1. Obtain $\boldsymbol{C}(1,1)$ from $\boldsymbol{D}(1,1), \mathbf{Z}(1,1)$ and $r_{3}$ with the following formula.

$$
\boldsymbol{C}(1,1)=\left[\boldsymbol{D}(1,1)+\boldsymbol{Z}(1,1)+r_{3}\right] \bmod 256
$$

Step 2. Let $j=2$ to $N$, then calculate $\boldsymbol{C}(1, j)$ for each $j$ with the following formula.

$$
\boldsymbol{C}(1, j)=[\boldsymbol{D}(1, j)+\boldsymbol{D}(1, j-1)+Z(1, j)] \bmod 256
$$

Step 3. Generate $\boldsymbol{C}(2,1)$ from $\boldsymbol{D}(2,1), \boldsymbol{Z}(2,1)$ and $\boldsymbol{D}(1, N)$ with the following formula.

$$
\boldsymbol{C}(2,1)=[\boldsymbol{D}(2,1)+\boldsymbol{D}(1, N)+\boldsymbol{Z}(2,1)] \bmod 256
$$

Step 4. Let $i=3$ to $M$, then calculate $\boldsymbol{C}(i, 1)$ for each $i$ with the following formula.

$$
\boldsymbol{C}(i, 1)=[\boldsymbol{D}(i, 1)+\boldsymbol{D}(i-1,1)+\boldsymbol{Z}(i, 1)] \bmod 256
$$

Step 5. Let $i=2$ to $M$, and $j=2$ to $N$, then calculate $C(i, j)$ for each combination of $i$ and $j$ with the following formula.

$$
\boldsymbol{C}(i, j)=[\boldsymbol{D}(i, j)+\boldsymbol{D}(i, j-1)+\boldsymbol{D}(i-1, j)+\boldsymbol{Z}(i, j)] \bmod 256
$$

After the above processing, one can get the cipher image $\boldsymbol{C}$ from $\boldsymbol{D}, \boldsymbol{Z}$ and $r_{3}$.

The above diffusion-II is reversible, and its inverse process recovers $\boldsymbol{D}$ from $\boldsymbol{C}, \boldsymbol{Z}$ and $r_{3}$. And the concrete steps of the inverse process of diffusion-II are as follows:

Step 1. Obtain $\boldsymbol{D}(1,1)$ from $\boldsymbol{C}(1,1), \mathbf{Z}(1,1)$ and $r_{3}$ with the following formula.

$$
\boldsymbol{D}(1,1)=\left[\boldsymbol{C}(1,1)+256 \times 2-\boldsymbol{Z}(1,1)-r_{3}\right] \bmod 256
$$

Step 2. Let $j=2$ to $N$, then calculate $\boldsymbol{D}(1, j)$ for each $j$ with the following formula.

$$
\boldsymbol{D}(1, j)=[\boldsymbol{C}(1, j)+256 \times 2-\boldsymbol{D}(1, j-1)-Z(1, j)] \bmod 256
$$

Step 3. Produce $\boldsymbol{D}(2,1)$ from $\boldsymbol{C}(2,1), \boldsymbol{Z}(2,1)$ and $\boldsymbol{D}(1, N)$ with the following formula.

$$
\boldsymbol{D}(2,1)=[\boldsymbol{C}(2,1)+256 \times 2-\boldsymbol{D}(1, N)-\boldsymbol{Z}(2,1)] \bmod 256
$$

Step 4. Let $i=3$ to $M$, then calculate $\boldsymbol{D}(i, 1)$ for each $i$ with the following formula.

$$
\boldsymbol{D}(i, 1)=[\boldsymbol{C}(i, 1)+256 \times 2-\boldsymbol{D}(i-1,1)-\boldsymbol{Z}(i, 1)] \bmod 256
$$

Step 5. Let $i=2$ to $M$, and $j=2$ to $N$, then calculate $\boldsymbol{D}(i, j)$ for each combination of $i$ and $j$ with the following formula.

$$
\boldsymbol{D}(i, j)=[\boldsymbol{C}(i, j)+256 \times 3-\boldsymbol{D}(i, j-1)-\boldsymbol{D}(i-1, j)-Z(i, j)] \bmod 256
$$

After the above processing, the matrix $\boldsymbol{D}$ is recovered from $\boldsymbol{C}$ with the help of $\boldsymbol{Z}$. 


\section{Simulation results}

The computer used is configured with Intel Core i7-4720HQ CPU and 8GB DDR3L memory. And C\# language based on Visual Studio 2017 Community Edition is used to program the proposed algorithm. Without losing generality, the 8-bit grayscale images of Lena, Baboon, Pepper, All-black image and All-white image are used in the simulation test (where, the images of Lena, Baboon and Pepper are from the USC-SIPI Image Database which is freely available at http://sipi.usc.edu/database/). And these images are all of size $256 \times 256$ pixels, and are shown in Figs. 4a-4e, respectively. In the test, the secret key $\boldsymbol{K}=\{255,138,130,97$, $14,17,172,88,194,103,0,137,234,71,208,26,26,243,207,231,15,113,130,61,252$, $217,9,243,21,254,80,143\}$ (in decimal format), the parameters of Chen's system $(a, b, c)$ $=(25,3,28)$, and the public key, i.e. the initial values of Chen's system, $\left(x_{0}, y_{0}, z_{0}\right)=(-8.319$, 12.0456, 36.789). Now, the proposed image encryption system is used to encrypt the plain images (as shown in Figs. 4a-4e, respectively), and the resultant cipher images are as shown in Figs. 4f-4j, respectively. Then the proposed image decryption system is used to decrypt the cipher images (as shown in Figs. $\mathbf{4 f - 4 j}$, respectively), and the recovered images are as shown in Figs. 4k-4o, respectively. The images in Figs. 4k-4o are exactly the same as the original plain images of Figs. 4a-4e, respectively, indicating that the proposed image cryptosystem works properly.

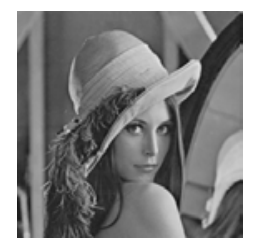

(a)

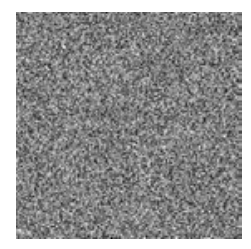

(f)

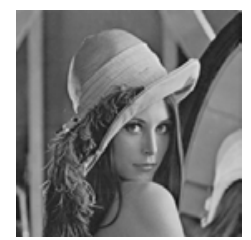

(k)

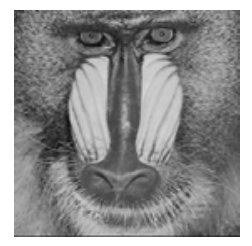

(b)

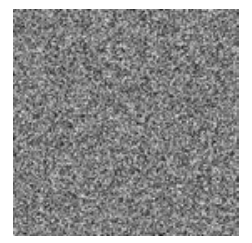

(g)

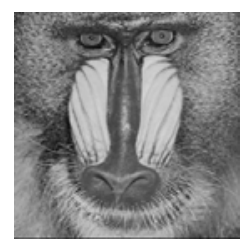

(l)

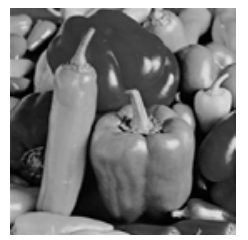

(c)

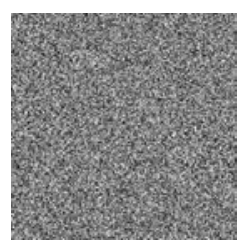

(h)

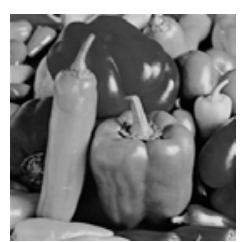

(m)

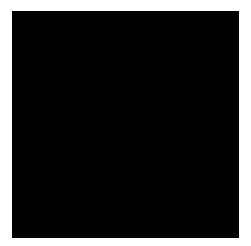

(d)

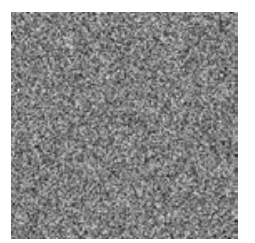

(i)

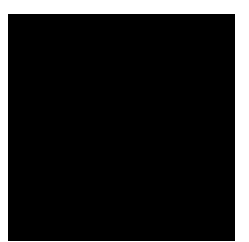

(n)

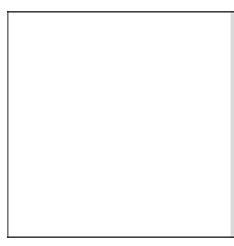

(e)

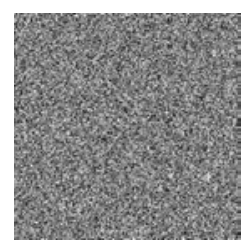

(j)

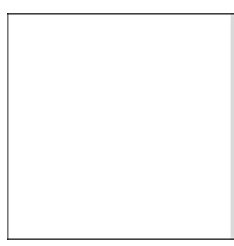

(o)

Fig. 4. Simulation results. (a)-(e) Plain images of Lena, Baboon, Pepper, All-black and All-white, respectively; (f)-(j) Cipher images of (a)-(e), respectively; (k)-(o) Recovered images of (f)-(j), respectively. 


\section{Security performance analysis}

\subsection{Key space}

Currently, the information security standard issued by NIST is AES, and its key length is 128, 192 or 256 bits. And NSA (National Security Agency) claimed that AES with key length of 192 or 256 bits can be used to encrypt the top secret level documents. Referring to the key length of AES, the key length of proposed image cryptosystem is 256 bits. Therefore, the key space of proposed cryptosystem is of size $2^{256}$, about $1.1579 \times 10^{77}$. Combining with the encryption/decryption speed in Section 5.4, the ability of proposed cryptosystem against the exhaustive attack will be discussed there.

In the proposed image cryptosystem, the initial values of Chen's system, i.e. $x_{0}, y_{0}$ and $z_{0}$, are open data and serve as the public key. Where, $x_{0} \in[-19.23,24.27], y_{0} \in[-21.03,27.47]$ and $z_{0} \in[6.86,44.00]$. And the step size respecting $x_{0}, y_{0}$ and $z_{0}$ is $10^{-13}$. Therefore, the size of public key space is about $7.8356 \times 10^{43}$, equivalent to the public key length being about 146 bits. The sensitivity of public key will be discussed in Section 5.3.4.

\subsection{Statistical characteristics analysis}

\subsubsection{Histogram analysis}

The histogram directly reflects the distribution of pixels of each gray value in the image. Without lose of generality, here take the plain images of Figs. 4a-4e and their corresponding cipher images of Figs. $\mathbf{4 f -} \mathbf{-} \mathbf{j}$ as examples to analyze the histogram difference between plain and cipher images. The histograms of plain images Figs. 4a-4e are shown in Figs. 5a-5e, respectively. And the histograms of cipher images Figs. $\mathbf{4 f - 4 j}$ are shown in Figs. $\mathbf{5 f}-\mathbf{5 j}$, respectively. Intuitively, the histograms of plain images have obvious fluctuations, while the histograms of cipher images are fairly flat. Therefore, there is distinguishable difference between them.

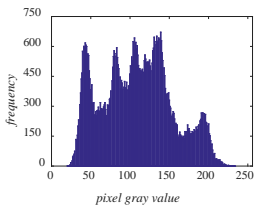

(a)

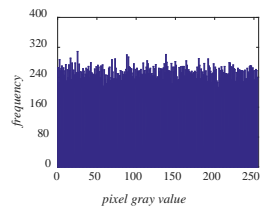

(f)

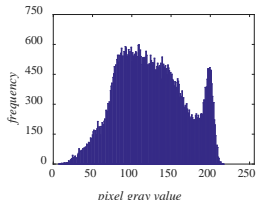

(b)

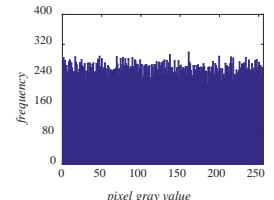

(g)

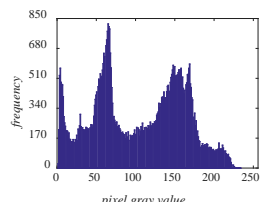

(c)

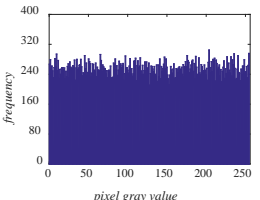

(h)

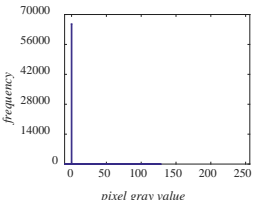

(d)

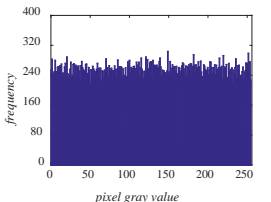

(i)

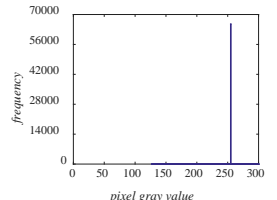

(e)

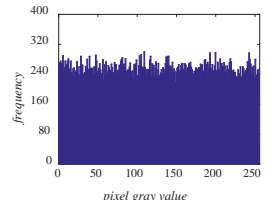

(j)

Fig. 5. Histograms. (a)-(e) Histograms of plain images Figs. 4a-4e, respectively;

(f)-(j) Histograms of cipher images Figs. $\mathbf{4 f - 4 j}$, respectively. 
The quantitative analysis on the difference between the histograms of plain and cipher images is described below.

It is well known that an 8-bit $M \times N$ sized grayscale image $\boldsymbol{I}$ characterized with uniform distribution has the mean $\mathrm{E}(\boldsymbol{I})$ and variance $\operatorname{Var}(\boldsymbol{I})$ as follows.

$$
\begin{aligned}
& \mathrm{E}(\boldsymbol{I})=\frac{1}{256} \sum_{i=0}^{255} i=127.5 \\
& \operatorname{Var}(\boldsymbol{I})=\frac{1}{256} \sum_{i=0}^{255} i^{2}-127.5^{2}=5.4613 \times 10^{3}
\end{aligned}
$$

However, for a certain 8-bit grayscale image $\boldsymbol{J}$ of size $M \times N$, whose pixel values are in unknown distribution, one can calculate its mean $\mathrm{E}(\boldsymbol{J})$ and variance $\operatorname{Var}(\boldsymbol{J})$ as follows.

$$
\begin{aligned}
& \mathrm{E}(J)=\frac{1}{M N} \sum_{i=1}^{M} \sum_{j=1}^{N} J(\mathrm{i}, \mathrm{j}) \\
& \operatorname{Var}(\boldsymbol{J})=\frac{1}{M N} \sum_{i=1}^{M} \sum_{j=1}^{N}[\boldsymbol{J}(\mathrm{i}, \mathrm{j})-\mathrm{E}(J)]^{2}
\end{aligned}
$$

According to Eqs. (45)-(46), the mean and variance of each histogram in Fig. 5 (i.e. the mean and variance of each image in Figs. $\mathbf{4 a - 4 j}$ ) are calculated, and the results are listed in Tables 3-4.

Table 3. The mean and variance of each histogram in Figs. 5a-5e

\begin{tabular}{|c|c|c|c|c|c|c|}
\hline & $\begin{array}{c}\text { Uniform } \\
\text { distribution }\end{array}$ & Fig. 5a & Fig. 5b & Fig. 5c & Fig. 5d & Fig. 5e \\
\hline \hline mean & 127.5 & 109.5 & 124.5 & 104.2 & 0 & 255 \\
\hline variance & $5.4613 \mathrm{e} 3$ & $2.0162 \mathrm{e} 3$ & $1.8339 \mathrm{e} 3$ & $3.2526 \mathrm{e} 3$ & 0 & 0 \\
\hline
\end{tabular}

Table 4. The mean and variance of each histogram in Figs. 5f-5j

\begin{tabular}{|c|c|c|c|c|c|c|}
\hline & $\begin{array}{c}\text { Uniform } \\
\text { distribution }\end{array}$ & Fig. 5f & Fig. 5g & Fig. 5h & Fig. 5i & Fig. 5j \\
\hline \hline mean & 127.5 & 127.4 & 126.9 & 127.5 & 127.8 & 127.1 \\
\hline variance & $5.4613 \mathrm{e} 3$ & $5.4587 \mathrm{e} 3$ & $5.4860 \mathrm{e} 3$ & $5.4768 \mathrm{e} 3$ & $5.4399 \mathrm{e} 3$ & $5.4585 \mathrm{e} 3$ \\
\hline
\end{tabular}

In Table 3, the mean and variance of each histogram in Figs. 5a-5e correspond sequentially to the mean and variance of each plain image in Figs. 4a-4e. Table 3 shows the differences between the plain images and uniformly distributed image in both mean and variance are significantly large, especially the minimum relative error of variance is greater than $40.44 \%$. However in Table 4, the mean and variance of each histogram in Figs. 5f-5j correspond sequentially to the mean and variance of each plain image in Figs. $\mathbf{4 f - 4 j}$. And Table 4 shows the differences between the cipher images and uniformly distributed image in both mean and variance are fairly small, and the maximum relative error of variance is less than $0.39 \%$. These demonstrate that the histograms of cipher images are very close to the uniform distribution, so the cipher images can resist the attack based on histogram analysis. 


\subsubsection{Correlation analysis}

In general, plain images have a strong correlation between adjacent pixels. While the image encryption algorithm will destroy the correlation seriously to make the adjacent pixels in ciphered images completely irrelevant. The correlation coefficient is usually used to measure the correlation between adjacent pixels of images. Assume that randomly select $K$ pairs of adjacent pixels from the image, and denote their values by $\left(u_{i}, v_{i}\right), i=1,2, \ldots, K$, then the correlation coefficient $r$ can be calculated with the following formula.

$$
r=\frac{\sum_{i=1}^{K}\left(u_{i}-\mathrm{E}(\boldsymbol{u})\right)\left(v_{i}-\mathrm{E}(\boldsymbol{v})\right)}{\sqrt{\sum_{i=1}^{K}\left(u_{i}-\mathrm{E}(\boldsymbol{u})\right)^{2} \sum_{i=1}^{K}\left(v_{i}-\mathrm{E}(\boldsymbol{v})\right)^{2}}}
$$

Where, $\mathrm{E}(\boldsymbol{u})$ and $\mathrm{E}(\boldsymbol{v})$ are separately the mean values of sequences $\left\{u_{i}\right\}$ and $\left\{v_{i}\right\}, i=1,2, \ldots, K$. From Eq. (47), when $r$ gets close to 1 or -1 , the correlation gets strong; when $r$ gets close to 0 , the correlation gets weak; when $r=0$, there is no correlation between $\boldsymbol{u}$ and $\boldsymbol{v}$.

Without loss of generality, take the plain images Figs. 4a-4e and their corresponding cipher images Figs. $\mathbf{4 f - 4 j}$ as examples to discuss the correlation issue. Here, let $K=2000$, and then from the tested images randomly select $K$ pairs of adjacent pixels in horizontal, vertical, diagonal and counter-diagonal directions respectively to calculate the correlation coefficients, and list the results in Table 5.

Table 5. Results of correlation coefficients.

\begin{tabular}{|c|c|c|c|c|c|}
\hline \multicolumn{2}{|c|}{ Image } & Horizontal & Vertical & Diagonal & Counter-diagonal \\
\hline \hline \multirow{2}{*}{ Lena } & Plain (Fig. 4a) & 0.96572 & 0.93161 & 0.91500 & 0.93761 \\
\cline { 2 - 6 } & Cipher (Fig. 4f) & 0.00070 & -0.01385 & 0.00020 & 0.01186 \\
\hline \multirow{2}{*}{ Baboon } & Plain (Fig. 4b) & 0.84340 & 0.87903 & 0.78298 & 0.80757 \\
\cline { 2 - 6 } & Cipher (Fig. 4g) & 0.00438 & -0.00261 & 0.00135 & 0.00883 \\
\hline \multirow{2}{*}{ Pepper } & Plain (Fig. 4c) & 0.97261 & 0.96512 & 0.92994 & 0.93890 \\
\cline { 2 - 6 } & Cipher (Fig. 4h) & -0.01168 & -0.00033 & 0.01072 & -0.01327 \\
\hline \multirow{2}{*}{ All-black } & Plain (Fig. 4d) & 1.00000 & 1.00000 & 1.00000 & 1.00000 \\
\cline { 2 - 6 } & Cipher (Fig. 4i) & -0.00592 & 0.03098 & -0.01783 & -0.04287 \\
\hline \multirow{2}{*}{ All-white } & Plain (Fig. 4e) & 1.00000 & 1.00000 & 1.00000 & 1.00000 \\
\cline { 2 - 6 } & Cipher (Fig. 4j) & 0.00142 & 0.01309 & -0.00976 & -0.00590 \\
\hline
\end{tabular}

In Table 5, the correlation coefficient of each plain image is close to 1 (and both All-black and All-white images are linearly correlated, and their correlation coefficients are both 1), i.e. the adjacent pixels in plain images have a strong positive correlation. However, the correlation coefficient of each cipher image is close to 0 , i.e. the adjacent pixels in cipher images are approximately uncorrelated. Therefore, Table 5 shows that the proposed image encryption algorithm completely removes the correlation between adjacent pixels in the original images. 
To visually compare the correlation characteristics between plain and cipher images, Fig. 6 illustrates the correlations of Fig. 4a (i.e. Lena) and Fig. 4 f (i.e. Lena's cipher image) both in horizontal direction. In Fig. 6a, 2000 pairs of selected adjacent pixels of Lena in horizontal direction are concentrated nearby the line of $y=x$, while, in Fig. $6 \mathbf{b}, 2000$ pairs of selected adjacent pixels of Lena's cipher image in horizontal direction are dispersed in the whole phase portrait. Thereby, Fig. 6 shows that the proposed image encryption algorithm effectively eliminates the correlation between adjacent pixels in the original images.

\subsubsection{Information entropy analysis}

The information entropy of an image reflects the uncertainty of the image information. For an 8-bit grayscale image with occurrence frequency $p(i)$ for gray value $i$, its information entropy $H$ is described as follows.

$$
H=-\sum_{i=0}^{255} p(i) \log _{2} p(i)
$$

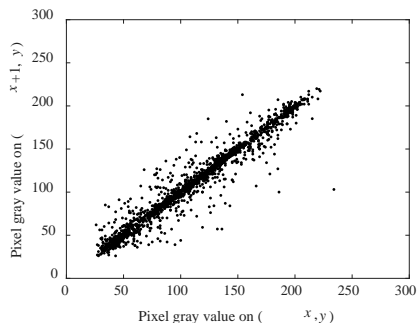

(a)

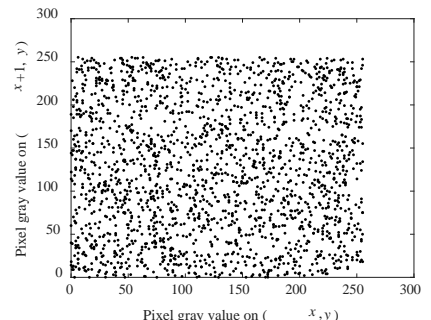

(b)

Fig. 6. The correlation between adjacent pixels in horizontal direction. (a) Case of Lena (Fig. 4a); (b) Case of Lena's cipher image (Fig. 4f)

Obviously, an 8-bit grayscale random image has the maximum uncertainty, and its information entropy gets to the maximum value of 8 bits. Generally, the information entropies of images with visual information are obviously less than 8 bits. Whereas the information entropies of cipher images are expected to be close to 8 bits. Here, the information entropies of the plain images Lena, Baboon, Pepper, All-black and All-white (see Figs. 4a-4e, respectively) and their corresponding cipher images (see Figs. 4f-4j, respectively) are separately calculated with Eq. (48), and the results are listed in Table 6.

Table 6. Information entropies of plain and cipher images (unit: bit)

\begin{tabular}{|c|c|c|c|c|c|}
\hline & Lena & Baboon & Pepper & All-black & All-white \\
\hline Plain image & 7.36848 & 7.35572 & 7.56456 & 0 & 0 \\
\hline Cipher image & 7.99734 & 7.99732 & 7.99663 & 7.99673 & 7.99735 \\
\hline
\end{tabular}


In Table 6, the information entropies of Lena, Baboon and Pepper are all less than 8 bits with the minimum relative error of 5.44\%, and the information entropies of All-black and All-white are both 0 . Whereas the information entropies of cipher images are all close to 8 bits with the maximum relative error of $0.042 \%$. These demonstrate that the cipher images are similar to noise-like images hiding the visual information perfectly.

\subsection{Sensitivity analysis}

In general, the sensitivity analysis of image cryptosystem includes three aspects, i.e. secret key sensitivity analysis, plaintext sensitivity analysis and cipher-text sensitivity analysis. Moreover, in the proposed cryptosystem, the initial values of Chen's system are regarded as public key, so the public key sensitivity analysis is also discussed in this part.

Two important indicators, known as NPCR and UACI [26], are widely used in the sensitivity analysis. NPCR (number of pixels change rate) measures the number of different pixels between two same sized images. And UACI (unified average changing intensity) measures the degree of difference between two same sized images. Assume that two $M \times N$ sized images are denoted by $\boldsymbol{I}_{1}$ and $\boldsymbol{I}_{2}$, respectively, then NPCR and UACI can be calculated by the following formulae.

$$
\operatorname{NPCR}\left(\boldsymbol{I}_{1}, \boldsymbol{I}_{2}\right)=\frac{1}{M N} \sum_{i=1}^{M} \sum_{j=1}^{N}\left|\operatorname{Sign}\left(\boldsymbol{I}_{1}(i, j)-\boldsymbol{I}_{2}(i, j)\right)\right| \times 100 \%
$$

Where, $\operatorname{Sign}(x)$ is the sign function, which returns 1 if $x>0,0$ if $x=0$, or -1 if $x<0$.

$$
\operatorname{UACI}\left(\boldsymbol{I}_{1}, \boldsymbol{I}_{2}\right)=\frac{1}{M N} \sum_{i=1}^{M} \sum_{j=1}^{N} \frac{\left|\boldsymbol{I}_{1}(i, j)-\boldsymbol{I}_{2}(i, j)\right|}{255-0} \times 100 \%
$$

For two random images of the same size, the expected values of NPCR and UACI are 99.6094\% and 33.4635\%, respectively. If an image is one of the Figs. 4a-4e and the other is a random image of the same size, the expected values of NPCR and UACI are as shown in

Table 7.

Table 7. The expected values of NPCR and UACI between a random image $\boldsymbol{R}$ and a plain image

\begin{tabular}{|c|c|c|c|c|c|}
\hline Index & $\begin{array}{c}\boldsymbol{R} \text { and Lana } \\
\text { (Fig. 4a) }\end{array}$ & $\begin{array}{c}\boldsymbol{R} \text { and Baboon } \\
\text { (Fig. 4b) }\end{array}$ & $\begin{array}{c}\boldsymbol{R} \text { and Pepper } \\
\text { (Fig. 4c) }\end{array}$ & $\begin{array}{c}\boldsymbol{R} \text { and } \\
\text { All-black (Fig. } \\
\text { 4d) }\end{array}$ & $\begin{array}{c}\boldsymbol{R} \text { and All-white } \\
\text { (Fig. 4e) }\end{array}$ \\
\hline \hline NPCR & $99.6094 \%$ & $99.6094 \%$ & $99.6094 \%$ & $99.6094 \%$ & $99.6094 \%$ \\
\hline UACI & $28.6850 \%$ & $27.9209 \%$ & $30.9134 \%$ & $50.0000 \%$ & $50.0000 \%$ \\
\hline
\end{tabular}

\subsubsection{Secret key sensitivity}

Secret key sensitivity investigates that the influence of tiny changes of secret keys on the ciphered images in encryption system or on the recovered images in decryption system. Excellent image cryptosystem must have a good sensitivity on secret keys, i.e. the tiny changes of each secret key will lead to completely different resultant images both in 
encryption and decryption systems.

The secret key sensitivity analysis includes two cases, i.e. the key sensitivity analysis of the encryption system and the key sensitivity analysis of the decryption system.

The process of key sensitivity analysis of the encryption system is as follows: Firstly, randomly generate a secret key of length 256 bits, and then randomly change its value by 1 bit to get a new key. Secondly, encrypt one plain image with these two keys to get two corresponding cipher images. Thirdly, calculate the values of NPCR and UACI between these two cipher images. Finally, repeat the test for 100 times to calculate the average values of NPCR and UACI, and then list the results in Table 8.

The process of key sensitivity analysis of the decryption system is as follows: Firstly, randomly generate a secret key of length 256 bits, denoted by $\boldsymbol{K}_{1}$. And then use $\boldsymbol{K}_{1}$ to encrypt a plain image $\boldsymbol{P}_{1}$ to get its corresponding cipher image $\boldsymbol{C}_{1}$. Secondly, randomly change the key $\boldsymbol{K}_{1}$ by 1 bit to get a new key, denoted by $\boldsymbol{K}_{2}$. Thirdly, use the key $\boldsymbol{K}_{2}$ to decrypt the cipher image $\boldsymbol{C}_{1}$ to get the recovered image, denoted by $\boldsymbol{P}_{2}$. Fourthly, calculate the values of NPCR and UACI between $\boldsymbol{P}_{1}$ and $\boldsymbol{P}_{2}$. Finally, repeat the test for 100 times to calculate the average values of NPCR and UACI, and then list the results in Table $\mathbf{9}$.

Here, without loss of generality, the images in Figs. 4a-4e (i.e. Lena, Baboon, Pepper, All-black and All-white images, respectively) are used as the tested images, and the initial values of Chen's system (i.e. public key) are set to $\left(x_{0}, y_{0}, z_{0}\right)=(-8.319,12.0456,36.789)$.

Table 8. Secret key sensitivity of the encryption system (unit: \%).

\begin{tabular}{|c|c|c|c|c|c|c|}
\hline Index & Lean & Baboon & Pepper & All-black & All-white & Theoretical \\
\hline \hline NPCR & 99.6067 & 99.6100 & 99.6108 & 99.6081 & 99.6044 & 99.6094 \\
\hline UACI & 33.4542 & 33.4541 & 33.4702 & 33.4598 & 33.4750 & 33.4635 \\
\hline
\end{tabular}

Table 9. Secret key sensitivity of the decryption system (unit: \%).

\begin{tabular}{|c|c|c|c|c|c|c|c|c|c|c|}
\hline \multirow{2}{*}{ Index } & \multicolumn{2}{|c|}{ Lena } & \multicolumn{2}{c|}{ Baboon } & \multicolumn{2}{c|}{ Pepper } & \multicolumn{3}{c|}{ All-black } & \multicolumn{2}{c|}{ All-white } \\
\cline { 2 - 32 } & Tested & Theor. & Tested & Theor. & Tested & Theor. & Tested & Theor. & Tested & Theor. \\
\hline \hline NPCR & 99.6097 & 99.6094 & 99.6107 & 99.6094 & 99.6087 & 99.6094 & 99.6082 & 99.6094 & 99.6071 & 99.6094 \\
\hline UACI & 28.6907 & 28.6850 & 27.9199 & 27.9209 & 30.9108 & 30.9134 & 50.0086 & 50.0000 & 49.9978 & 50.0000 \\
\hline
\end{tabular}

Tables 8-9 show the tested values of NPCR and UACI are both close to their theoretical values with the maximum relative error of $0.034 \%$ in both encryption and decryption systems, thereby indicating that the proposed cryptosystem possesses a strong sensitivity on secret keys.

\subsubsection{Plaintext sensitivity}

The plaintext sensitivity measures the degree of influence of tiny changes in plain images on the image encryption system. Excellent image encryption system must have a good plaintext 
sensitivity, i.e. the tiny changes of each plain image inputted into the image encryption system will cause two completely different encrypted images.

Here, the images in Figs. 4a-4e (i.e. Lena, Baboon, Pepper, All-black and All-white images, respectively) are taken as examples in the plaintext sensitivity test. Firstly, make slight change for each plain image (i.e. change its one random pixel's value by 1) to obtain one pair of images with only one different pixel. Secondly, encrypt these two images with the proposed image encryption system (using the identical secret key) to obtain their corresponding cipher images. Thirdly, use these two resultant images to calculate the values of NPCR and UACI. Finally, repeat this test for 100 times to calculate the average values of NPCR and UACI, which are listed in Table 10. In each test, the secret key is randomly selected, and the initial values of Chen's system (i.e. public key) are set to $\left(x_{0}, y_{0}, z_{0}\right)=(-8.319$, 12.0456, 36.789).

Table 10. Results of plaintext sensitivity test (unit: \%).

\begin{tabular}{|c|c|c|c|c|c|c|}
\hline Index & Lean & Baboon & Pepper & All-black & All-white & Theoretical \\
\hline \hline NPCR & 99.6078 & 99.6017 & 99.6092 & 99.6075 & 99.6087 & 99.6094 \\
\hline UACI & 33.4476 & 33.4395 & 33.4552 & 33.4653 & 33.4598 & 33.4635 \\
\hline
\end{tabular}

As can be seen in Table 10, the calculated results of NPCR and UACI are fairly close to their theoretical values with the maximum relative error of $0.072 \%$, thereby showing that the proposed image encryption system has a strong plaintext sensitivity.

\subsubsection{Cipher-text sensitivity}

The plaintext sensitivity measures the sensitivity of image encryption system, while the cipher-text sensitivity measures the sensitivity of image decryption system. Excellent image decryption system must have a strong cipher-text sensitivity, i.e. the tiny changes in cipher images will lead to that the decrypted images completely different form the original plain images.

Here, take the images of Figs. 4a-4e (i.e. Lena, Baboon, Pepper, All-black and All-white images, respectively) as the tested images. Firstly, encrypt the images of Figs. 4a-4e with the proposed image encryption system to get their corresponding cipher images. Secondly, make slight change for each cipher image (i.e. change its one random pixel's value by 1) to obtain one pair of images with only one different pixel. Thirdly, decrypt this pair of images with the proposed image decryption system (using the same secret key as that used in the encryption system) to obtain the original plain image and one decrypted image. Finally, use these two resultant images to calculate the values of NPCR and UACI. Repeat this test for 100 times to calculate the average values of NPCR and UACI, and then list the results in Table 11. Note that in each test the secret key is randomly selected, and the initial values of Chen's system (i.e. public key) are set to $\left(x_{0}, y_{0}, z_{0}\right)=(-8.319,12.0456,36.789)$. 
Table 11. Results of cipher-text sensitivity test (unit: \%).

\begin{tabular}{|c|c|c|c|c|c|c|c|c|c|c|}
\hline \multirow{2}{*}{ Index } & \multicolumn{2}{|c|}{ Lena } & \multicolumn{2}{c|}{ Baboon } & \multicolumn{2}{c|}{ Pepper } & \multicolumn{3}{c|}{ All-black } & \multicolumn{2}{c|}{ All-white } \\
\cline { 2 - 12 } & Tested & Theor. & Tested & Theor. & Tested & Theor. & Tested & Theor. & Tested & Theor. \\
\hline \hline NPCR & 99.6069 & 99.6094 & 99.6083 & 99.6094 & 99.6107 & 99.6094 & 99.6130 & 99.6094 & 99.6062 & 99.6094 \\
\hline UACI & 28.6877 & 28.6850 & 27.9256 & 27.9209 & 30.9091 & 30.9134 & 50.0209 & 50.0000 & 49.9903 & 50.0000 \\
\hline
\end{tabular}

In Table 11, the calculated values of NPCR and UACI are approximately equal to their theoretical values with the maximum relative error of $0.072 \%$, which shows that the proposed image decryption system has a strong cipher-text sensitivity.

\subsubsection{Public key sensitivity}

In the proposed image cryptosystem, Chen's system is used to generate the key streams. The three initial values of Chen's system $\left(x_{0}, y_{0}, z_{0}\right)$ are open and serve as the public key. The ranges of $\left(x_{0}, y_{0}, z_{0}\right)$ are slightly smaller than the state space of Chen's system. Here, let $x_{0} \in[-19.23,24.27], y_{0} \in[-21.03,27.47], z_{0} \in[6.86,44.00]$, and their step sizes be all $10^{-13}$.

In the public key sensitivity test, randomly generate a public key (i.e. $\left(x_{0}, y_{0}, z_{0}\right)$ ), and change one element of the public key by $10^{-13}$ while keeping the other two elements unchanged to obtain the slightly changed public key. Then, use these two slightly different public keys separately as the initial values of Chen's system to generate two key streams (i.e. two sets of $\left.\left\{\boldsymbol{X}, \boldsymbol{Y}, \boldsymbol{Z}, r_{1}, r_{2}, r_{3}\right\}\right)$. Employ the two key streams in the encryption/decryption system to get two resultant images, and calculate the values of NPCR and UACI between the resultant images. Finally, repeat the test for 100 times to get the average values of NPCR and UACI, and list the results in Tables 12-13. Note that in each test a random secret key is used.

Table 12. Public key sensitivity of image encryption system (unit: \%).

\begin{tabular}{|c|c|c|c|c|c|c|c|}
\hline \multicolumn{2}{|c|}{ Index } & Lean & Baboon & Pepper & All-black & All-white & Theoretical \\
\hline \hline \multirow{2}{*}{$x_{0}$} & NPCR & 99.6107 & 99.6117 & 99.6095 & 99.6117 & 99.6096 & 99.6094 \\
\cline { 2 - 8 } & UACI & 33.4678 & 33.4638 & 33.4670 & 33.4538 & 33.4639 & 33.4635 \\
\hline \multirow{2}{*}{$y_{0}$} & NPCR & 99.6085 & 99.6071 & 99.6105 & 99.6048 & 99.6100 & 99.6094 \\
\cline { 2 - 8 } & UACI & 33.4735 & 33.4506 & 33.4627 & 33.4738 & 33.4638 & 33.4635 \\
\hline \multirow{2}{*}{$z_{0}$} & NPCR & 99.6125 & 99.6118 & 99.6100 & 99.6102 & 99.6098 & 99.6094 \\
\cline { 2 - 8 } & UACI & 33.4568 & 33.4704 & 33.4637 & 33.4534 & 33.4597 & 33.4635 \\
\hline
\end{tabular}


Table 13. Public key sensitivity of image decryption system (unit: \%).

\begin{tabular}{|c|c|c|c|c|c|c|c|c|c|c|c|}
\hline \multirow{2}{*}{\multicolumn{2}{|c|}{ Index }} & \multicolumn{2}{|c|}{ Lena } & \multicolumn{2}{|c|}{ Baboon } & \multicolumn{2}{|c|}{ Pepper } & \multicolumn{2}{|c|}{ All-black } & \multicolumn{2}{|c|}{ All-white } \\
\hline & & Tested & Theor. & Tested & Theor. & Tested & Theor. & Tested & Theor. & Tested & Theor. \\
\hline \multirow{2}{*}{$x_{0}$} & NPCR & 99.6147 & 99.6094 & 99.6068 & 99.6094 & 99.6133 & 99.6094 & 99.6122 & 99.6094 & 99.6104 & 99.6094 \\
\hline & UACI & 28.6842 & 28.6850 & 27.9178 & 27.9209 & 30.8929 & 30.9134 & 50.0157 & 50.0000 & 50.0110 & 50.0000 \\
\hline \multirow{2}{*}{$y_{0}$} & NPCR & 99.6022 & 99.6094 & 99.6120 & 99.6094 & 99.6051 & 99.6094 & 99.6052 & 99.6094 & 99.6077 & 99.6094 \\
\hline & UACI & 28.6860 & 28.6850 & 27.9270 & 27.9209 & 30.9177 & 30.9134 & 49.9780 & 50.0000 & 49.9840 & 50.0000 \\
\hline \multirow{2}{*}{$Z_{0}$} & NPCR & 99.6106 & 99.6094 & 99.6075 & 99.6094 & 99.6061 & 99.6094 & 99.6120 & 99.6094 & 99.6052 & 99.6094 \\
\hline & UACI & 28.6843 & 28.6850 & 27.9226 & 27.9209 & 30.9039 & 30.9134 & 49.9843 & 50.0000 & 49.9942 & 50.0000 \\
\hline
\end{tabular}

Tables 12-13 reflect the degree of influence of small changes of public key on the encryption and decryption systems. In Tables 12-13, the calculated values of NPCR and UACI are very close to their theoretical values, i.e. the proposed image cryptosystem have a strong sensitivity on public keys. These also say that one can arbitrarily choose a public key in the public key space.

\subsection{Encryption and decryption speed}

The computer in Section 4 and C\# program are used to test the encryption and decryption speed of proposed image cryptosystem. If the time of encrypting/decrypting an image of size $M \times N$ is denoted by $t_{1}$ or $t_{2}$ seconds respectively, then the encryption/decryption speed is $8 M N / t_{1}$ or $8 M N / t_{2}$ bps respectively. If the time of generating key streams for $M \times N$ sized image is denoted by $t_{3}$ seconds, then the speed of key stream generating is $8 M N / t_{3}$ bps. Here, take the 8-bit grayscale image Lena of size 256×256 pixels as an example, and list the tested results in Table 14.

Table 14. Encryption/Decryption speed of proposed system.

\begin{tabular}{|c|c|c|c|c|c|}
\hline & $\begin{array}{c}\text { Key stream } \\
\text { generator }\end{array}$ & $\begin{array}{c}\text { Encryption } \\
\text { process }\end{array}$ & $\begin{array}{c}\text { Decryption } \\
\text { process }\end{array}$ & $\begin{array}{c}\text { Key stream } \\
\text { generator + } \\
\text { encryption } \\
\text { process }\end{array}$ & $\begin{array}{c}\text { Key stream } \\
\text { generator }+ \\
\text { decryption } \\
\text { process }\end{array}$ \\
\hline \hline Time (ms) & 27.309 & 14.972 & 16.026 & 42.281 & 43.335 \\
\hline $\begin{array}{c}\text { Speed } \\
\text { (Mbps) }\end{array}$ & - & 35.018 & 32.715 & 12.400 & 12.098 \\
\hline
\end{tabular}

In general, once the secret key is established between two communication sides, it will be used for a period of time, so the encryption/decryption speed of proposed cryptosystem in Table 14 is referred to 35.018 Mbps or 32.715 Mbps, respectively. While the encryption/decryption speed of proposed system containing the key stream generator is separately $12.400 \mathrm{Mbps}$ or $12.098 \mathrm{Mbps}$ and is equivalent to the speed of one-time pad, 
which is the speed for the brute-force attacking the system once. Therefore, if the computer used in Section 4 is employed to crack the proposed encryption/decryption system by the brute-force attack method, half of the key space will be tried out and at least $7.7622 \times 10^{67}$ or $7.9557 \times 10^{67}$ years will be required respectively. So, the proposed system can combat the exhaustive attack.

\subsection{Comparative analysis}

In order to prove that the proposed image cryptosystem has excellent performance, the proposed system is compared with AES (in CBC mode) and those systems described in Refs. [27-29]. All these systems can produce the cipher images with excellent statistical characteristics, so the compared items are limited to the encryption/decryption speed, secret key sensitivity, plaintext sensitivity and cipher-text sensitivity. And the comparison results are listed in Table 15. Note that (1) the 8-bit grayscale image Lena of size $256 \times 256$ pixels is used; (2) the speed of each image encryption/decryption system excludes that of key stream generator; (3) the scheme of AES uses a secret key of length 256 bits [25]; (4) the scheme in [27] uses a secret key of length 256 bits; (5) the scheme in [28] uses three double-precision floating-point numbers as the secret key (equivalent to the key length of 140 bits); (6) the scheme in [29] uses four double-precision floating-point numbers as the secret key (equivalent to the key length of 186 bits); (7) the values of NPCR and UACI are the average values of 100 tests.

Table 15. Results of comparative analysis

\begin{tabular}{|c|c|c|c|c|c|c|c|c|c|c|}
\hline \multirow{3}{*}{$\begin{array}{c}\text { Image } \\
\text { cryptosystem }\end{array}$} & \multirow{3}{*}{$\begin{array}{c}\text { Enc. } \\
\text { speed } \\
\text { (Mbps) }\end{array}$} & \multirow{3}{*}{$\begin{array}{c}\text { Dec. } \\
\text { speed } \\
\text { (Mbps) }\end{array}$} & \multicolumn{4}{|c|}{ Secret key sensitivity (\%) } & \multirow{2}{*}{\multicolumn{2}{|c|}{$\begin{array}{c}\text { Plaintext } \\
\text { sensitivity (\%) }\end{array}$}} & \multirow{2}{*}{\multicolumn{2}{|c|}{$\begin{array}{c}\text { Cipher-text } \\
\text { sensitivity (\%) }\end{array}$}} \\
\hline & & & \multicolumn{2}{|c|}{ Enc. process } & \multicolumn{2}{|c|}{ Dec. process } & & & & \\
\hline & & & NPCR & UACI & NPCR & UACI & NPCR & UACI & NPCR & UACI \\
\hline Theoretical & - & - & 99.6094 & 33.4635 & 99.6094 & 28.6850 & 99.6094 & 33.4635 & 99.6094 & 28.6850 \\
\hline AES & 7.2496 & 6.6223 & 99.6095 & 33.4564 & 99.5996 & 28.6531 & 99.6049 & 33.4798 & 99.6079 & 28.6853 \\
\hline Ref. [27] & 36.587 & 35.223 & 75.7019 & 25.4295 & 86.6635 & 24.8754 & 99.5988 & 33.0621 & 0.0245 & 0.0001 \\
\hline Ref. [28] & 137.862 & 2.967 & 98.2652 & 33.0074 & 98.2919 & 27.8255 & 0.0015 & 0.0005 & 0.0015 & 0.0004 \\
\hline Ref. [29] & 0.068 & 0.068 & 99.6170 & 33.4531 & 99.4099 & 22.7641 & 99.5879 & 33.4334 & 99.6132 & 28.5904 \\
\hline Proposed & 35.018 & 32.715 & 99.6067 & 33.4542 & 99.6097 & 28.6907 & 99.6078 & 33.4476 & 99.6069 & 28.6877 \\
\hline
\end{tabular}

(Note: In Table 15, 'Enc.' and 'Dec.' are separately the abbreviations of 'Encryption' and 'Decryption'. )

From Table 15, the encryption and decryption speeds of AES are separately 7.2496 and 6.6223 Mbps. If the encryption and decryption speeds of AES are taken as two thresholds, the speed of scheme in [29] is too slow, and the decryption speed of scheme in [28] is also slow (although its encryption speed is very fast). The speeds of both proposed scheme and the scheme in [27] are faster than the thresholds so as to meet the speed requirement. 
In fact, the scheme in [28] is essentially a one-time pad system using RSA technology to complete the secret key exchange for each secure communication. So the scheme in [28] has no plaintext and cipher-text sensitivities as shown in Table 15, and cannot resist the known/chosen plaintext attacks. From Table 15, the scheme in [27] has no cipher-text sensitivity, which is a defect in resisting the known/chosen cipher-text attacks. The scheme in [29] uses SHA-3 technology and possesses excellent sensitivities as shown in Table 15, but with very slow encryption/decryption speed, which limits its application. Like the scheme in [29], the proposed scheme also possesses excellent sensitivities.

So in the five schemes listed in Table 15, only the proposed scheme has the best comprehensive performance.

\section{Conclusion}

Unlike most of recent image cryptosystems based on confusion-diffusion architecture, this paper proposed an image cryptosystem based on substitution-diffusion architecture. The substitution algorithm "removes" the information of original image from the encrypted images, so that the useful information of plain image can be hidden better. Combining the substitution algorithm with the diffusion processes, the proposed scheme makes the changes of each pixel in the plain image affect the whole cipher image. Simulation results show that the proposed image cryptosystem has the characteristics of fast encryption/decryption speed and strong sensitivities. So the proposed system can be used as an alternative scheme for the real network communication.

The substitution technology is widely used in data cryptography. This paper used the S-Box of AES to carry out substitution operation and achieved satisfactory security results. In the proposed scheme, two diffusion processes are used to get the excellent plain/cipher-text sensitivity, which cost a lot of time. The future work will focus on studying the image scheme with new substitution algorithm and only one diffusion process, so as to further improve the encryption/decryption speed without affecting the system security.

\section{Acknowledgement}

This work was fully supported by the National Science Foundation of China (Grant Nos. 61762043, 61562035 and 61702238), the Natural Science Foundation of Jiangxi Province, China (Grant No. 20161BAB202058), and the Science and Technology Project of Education Department of Jiangxi Province, China (Grant No. GJJ160426). 


\section{References}

[1] Y. Zhou, L. Bao and C. L. P. Chen, "A new 1D chaotic system for image encryption,” Signal Processing, vol. 97, no. 7, pp. 172-182, April 2014. Article (CrossRef Link).

[2] X. Li, G. Zhang and X. Zhang, "Image encryption algorithm with compound chaotic maps," Journal of Ambient Intelligence and Humanized Computing, vol. 6, no. 5, pp. 563-570, May 2015. Article (CrossRef Link).

[3] A. Akif, M. Irene, P. Ihsan and S. Vaidyanathan, "A new four-scroll chaotic attractor and its engineering applications,” Optik, vol. 127, no. 13, pp. 5491-5499, July 2016.

Article (CrossRef Link).

[4] E. Chen, L. Min and G. Chen, "Discrete chaotic systems with one-line equilibria and their application to image encryption," International Journal of Bifurcation and Chaos, vol. 27, no. 3, pp. 1750046 (17 pages), March 2017. Article (CrossRef Link).

[5] N. B. Slimane, K. Bouallegue and M. Machhout, "Designing a multi-scroll chaotic system by operating Logistic map with fractal process," Nonlinear Dynamics, vol. 88, no. 3, pp. 1655-1675, March 2017. Article (CrossRef Link).

[6] T. Sivakumar and R. Venkatesan, "A novel image encryption using calligraphy based scan method and random number," KSII Transactions on Internet \& Information Systems, vol. 9, no. 6, pp. 2317-2337, June 2015. Article (CrossRef Link).

[7] J. S. A. E. Fouda, J. Y. Effa, S. L. SamratL and M. Ali, "A fast chaotic block cipher for image encryption,” Communications in Nonlinear Science \& Numerical Simulation, vol. 19, no. 3, pp. 578-588, March 2014. Article (CrossRef Link).

[8] X. Wang and D. Xu, "A novel image encryption scheme using chaos and Langton's Ant cellular automaton,” Nonlinear Dynamics, vol. 79, no. 4, pp. 2449-2456, March 2015.

Article (CrossRef Link).

[9] Y. Zhang, "A chaotic system based image encryption scheme with identical encryption and decryption algorithm," Chinese Journal of Electronics, vol. 26, no. 5, pp. 1022-1031, October 2017. Article (CrossRef Link).

[10] P. Li and Y. Zhao, "A simple encryption algorithm for quantum color image," International Journal of Theoretical Physics, vol. 56, no. 6, pp. 1961-1982, June 2017.

Article (CrossRef Link).

[11] C. Li, "Cracking a hierarchical chaotic image encryption algorithm based on permutation," Signal Processing, vol. 118, no. C, pp. 203-210, January 2016. Article (CrossRef Link).

[12] J. A. Jolfaei, X. Wu and V. Muthukkumarasamy, "On the security of permutation-only image encryption schemes," IEEE Transactions on Information Forensics and Security, vol. 11, no. 2, pp. 235-246, February 2016. Article (CrossRef Link).

[13] D. S. Laiphrakpam and M. S. Khumanthem, "Cryptanalysis of symmetric key image encryption using chaotic Rossler system,” Optik, vol. 135, no. 4, pp. 200-209, April 2017.

Article (CrossRef Link). 
[14] B. Norouzi and S. Mirzkuchaki, "Breaking a novel image encryption scheme based on an improper fractional order chaotic system,” Multimedia Tools \& Applications, vol. 76, no. 2, pp. 1817-1826, February 2017. Article (CrossRef Link).

[15] W. Wen, Y. Zhang, M. Su, R. Zhang, J. Chen and M. Li, "Differential attack on a hyper-chaos-based image cryptosystem with a classic bi-modular architecture," Nonlinear Dynamics, vol. 87, no. 1, pp. 383-390, January 2017. Article (CrossRef Link).

[16] Y. Zhang and Y. Tang, "A plaintext-related image encryption algorithm based on chaos," Multimedia Tools and Applications, vol. 77, no. 6, pp. 6647-6669, March 2018.

Article (CrossRef Link).

[17] X. Chai, Z. Gan, K. Yang, Y. Chen and X. Liu, "An image encryption algorithm based on the memristive hyperchaotic system, cellular automata and DNA sequence operations," Signal Processing: Image Communication, vol. 52, no. C, pp. 6-19, March 2017. Article (CrossRef Link).

[18] A. V. Diaconu, "Circular inter-intra pixels bit-level permutation and chaos-based image encryption,” Information Sciences, vol. 355-356, pp. 314-327, August 2016.

Article (CrossRef Link).

[19] H. Fan and M. Li, "Cryptanalysis and improvement of chaos-based image encryption scheme with circular inter-intra-pixels bit-level permutation," Mathematical Problems in Engineering, vol. 2017, pp. 1-11, July 2017. Article (CrossRef Link).

[20] W. Zhang, H. Yu, Y. Zhao and Z. Zhu, "Image encryption based on three-dimensional bit matrix permutation,” Signal Processing, vol. 118, no. 1, pp. 36-50, January 2016.

Article (CrossRef Link).

[21] J. Wu, X. Liao and B. Yang, "Cryptanalysis and enhancements of image encryption based on three-dimensional bit matrix permutation,” Signal Processing, vol. 142, no. 1, pp. 292-300, January 2018. Article (CrossRef Link).

[22] X. Wang and H. Zhang, "A novel image encryption algorithm based on genetic recombination and hyper-chaotic systems,” Nonlinear Dynamics, vol. 83, no. 1-2, pp. 333-346, February 2016. Article (CrossRef Link).

[23] T. Ueta and G. Chen, "Bifurcation analysis of Chen's equation," International Journal of Bifurcation \& Chaos, vol. 10, no. 8, pp. 1917-1931, August 2000. Article (CrossRef Link).

[24] National Institute of Standards and Technology, "Federal Information Processing Standard FIPS PUB 140-2, Security requirements for cryptographic modules,” 2002. Article (CrossRef Link)

[25] J. Daemen and V. Rijmen, "The design of Rijndael: AES - the Advanced Encryption Standard," Springer-Verlag, Berlin, 2002.

[26] G. Chen, Y. Mao and C. K. Chui, "A symmetric image encryption scheme based on 3D chaotic cat maps,” Chaos Solitons \& Fractals, vol. 21, no. 3, pp. 749-761, July 2004. Article (CrossRef Link). 
[27] Z. Hua, Y. Zhou, C. Pun and C. L. P. Chen, "2D Sine Logistic modulation map for image encryption,” Information Sciences, vol. 297, no. C, pp. 80-94, March 2015.

Article (CrossRef Link).

[28] Ünal Çavuşoğlu, S. Kaçar, I. Pehlivan I and A. Zengin, "Secure image encryption algorithm design using a novel chaos based S-Box,” Chaos Solitons \& Fractals, vol. 95, no. 2, pp. 92-101, February 2017. Article (CrossRef Link).

[29] G. Ye, H. Zhao and H. Chai, "Chaotic image encryption algorithm using wave-line permutation and block diffusion,” Nonlinear Dynamics, vol. 83, no. 4, pp. 2067-2077, April 2016.

Article (CrossRef Link).

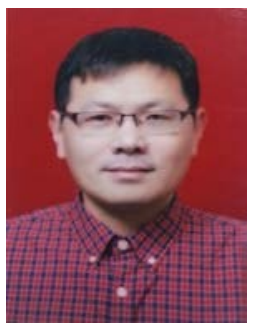

Yong Zhang received the MS degree in communication and information systems and the $\mathrm{PhD}$ degree in circuits and systems both from University of Electronic Science and Technology of China (UESTC), in 2003 and in 2006 respectively. Currently, he is an associate professor at School of Software and Communication Engineering in Jiangxi University of Finance and Economics (JXUFE) in China. His research interests focus on the area of information security and quantum information.

E-mail: zhangyong@jxufe.edu.cn

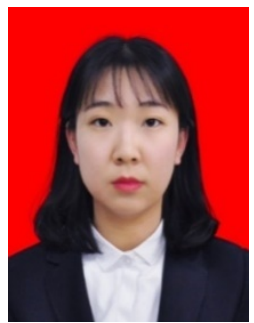

Xiaoyang Jia received the Bachelor's degree in detection guidance and control technology and the MS degree in optical engineering both from Changchun University of Science and Technology (CUST) in China, in 2014 and in 2017 respectively. She serves as an associate lecturer in Changchun China Optical Science \& Technology Museum. Her research interests focus on the area of information security and space laser communication. E-mail: jiaxiaoyang_cc@126.com 